\title{
Treatment of Psoriasis After Initiation of Nivolumab Therapy for Metastatic Malignant Melanoma: An Ancient Drug Revisited
}

\author{
(D) Gökhan Okan¹, (1) Kezban Nur PilancI², (1) Cuyan Demirkesen ${ }^{3}$ \\ 1Private Dermatologist, Istanbul, Turkey \\ 2Memorial Bahcelievler Hospital, Clinic of Oncology, Istanbul, Turkey \\ ${ }^{3}$ Acibadem University Faculty of Medicine, Department of Pathology, Istanbul, Turkey
}

Keywords: Checkpoint inhibitors, Nivolumab, Psoriasis, Melanoma, Sulfasalazine

\section{Dear Editor,}

Checkpoint inhibition can cause various immune-related adverse events in any organ but skin toxicity occurs most frequently [1]. We report de novo development of psoriasis vulgaris in a patient receiving nivolumab for treatment of metastatic melanoma which was controlled with sulfasalazine (SSZ).

A 69-year-old male was initiated with nivolumab $3 \mathrm{mg} / \mathrm{kg}$ every two weeks for metastatic BRAF-negative melanoma of the back. The patient's medical history was notable for arterial hypertension and dyslipidemia for which he has been medically treated. There was no personal or family history of psoriasis. One week after the fourth cycle of nivolumab, asymptomatic, sharply bordered, erythematous and scaly plaques were seen on the the anterolateral aspects of shins, dorsa of hands, feet, scalp, and trunk (Figure 1a). A skin biopsy revealed psoriasiform epidermal hyperplasia with prominent orthohyperkeratosis, and mounds of parakeratosis, containing neutrophils. The suprapapillary epidermis was thinned, and there were collections of neutrophils in the spinous layers which were consistent with psoriasis (Figure 1b, 1c). The patient had psoriasis area and severity index score of 12 . He did not have psoriatic arthritis. The patient's melanoma improved with nivolumab therapy but the skin lesions increased. As the patient was refractory to topical therapies, he was switched to acitretin but experienced severe side effects (hyperlipidemia), so therapy

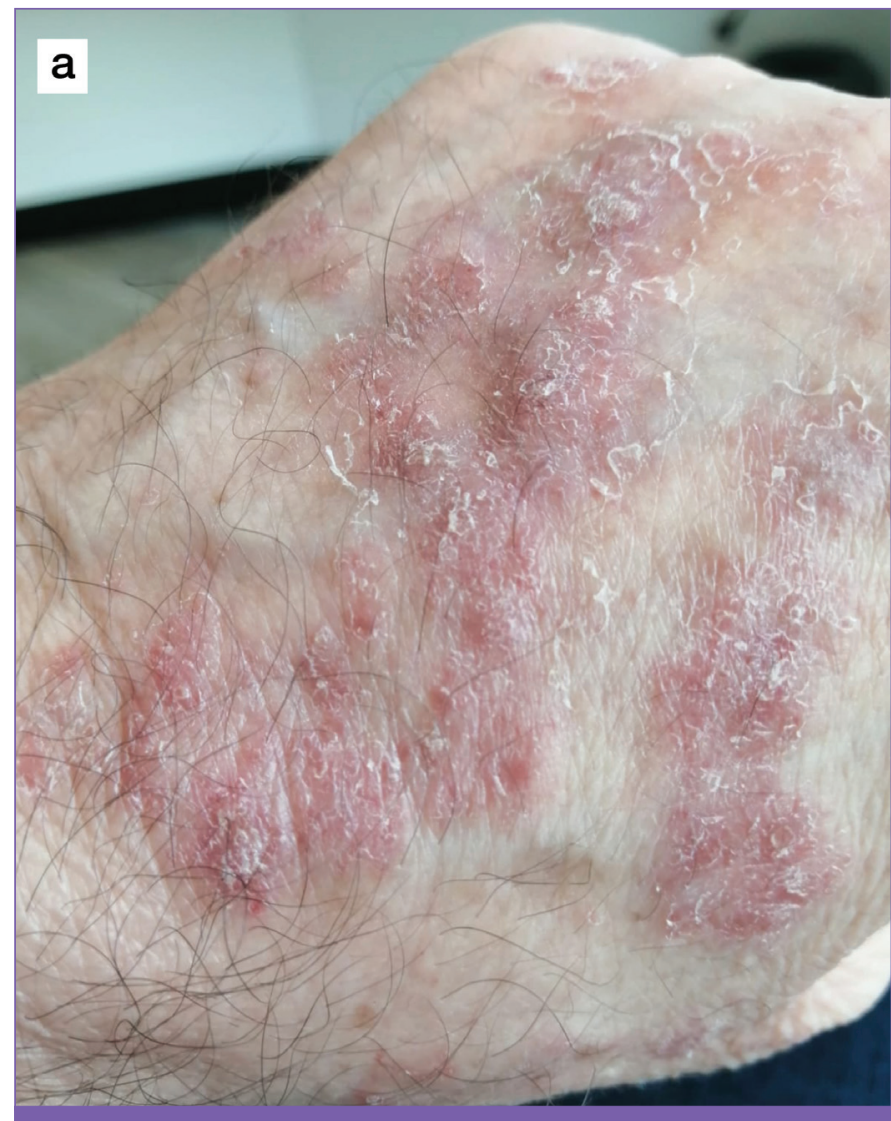

Figure 1. a) Psoriatic lesions on the hands 

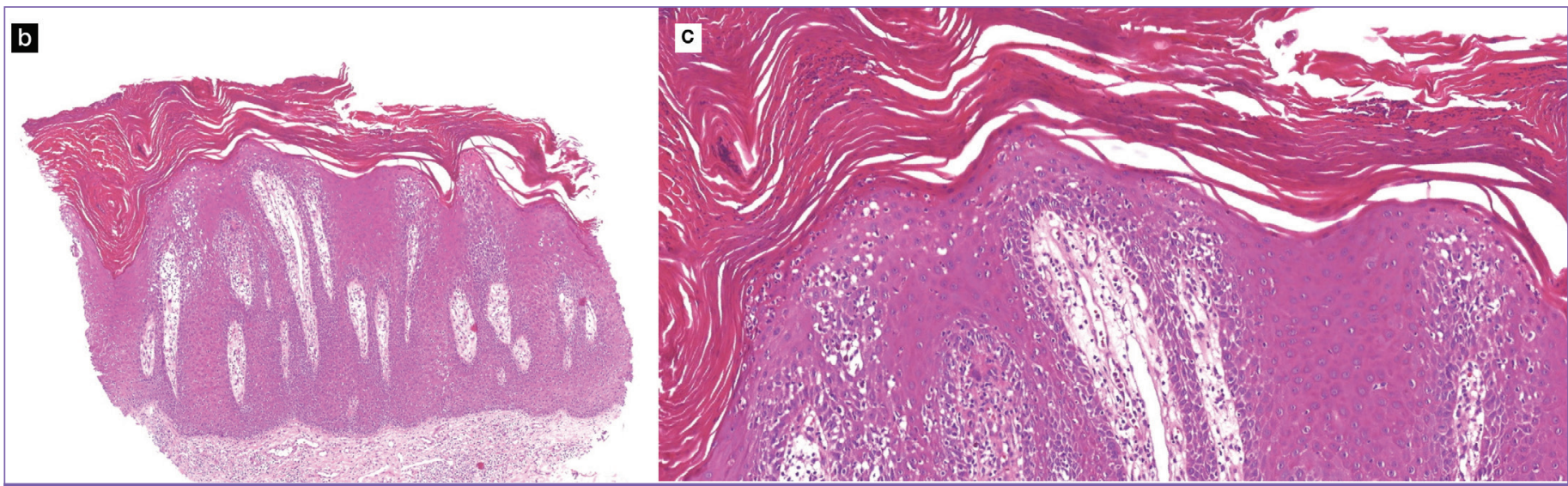

Figure 1. b, c) Psoriasiform epidermal hyperplasia with mounds of parakeratosis and collections of neutrophils in the upper spinous layers (hematoxylin and eosin $\AA \sim 40$; hematoxylin and eosin $\AA \sim 200$ )

was discontinued at the end of two months. Methotrexate was initiated, but led to liver toxicity a short period of time. Due to numerous drug failures and the high cost of biologic therapies, we decided to start SSZ (2 gr/day). SSZ therapy controlled the psoriatic lesions with no side effects. Currently, the patient is in remission and his skin lesions are under control.

Nivolumab can give rise to a variety of cutaneous side effects that may influence decisions about the therapy. In our patient psoriatic lesions developed nine weeks after nivolumab therapy with no prior history of psoriasis. Increased Th1 and Th17 responses induced by nivolumab are important for its' an antitumor effect but may unmask a psoriatic reaction in a predisposed individual [2].

SSZ induces few side effects and has been used to treat of psoriasis and psoriatic arthritis with variable success. SSZ has been shown to decrease cytokine release, including tumor necrosis factor-alpha (TNF- $\alpha$ ) [3]. Blocking TNF- $\alpha$ has been demonstrated to prevent checkpoint inhibitor induced colitis in a genetically susceptible animal model [4]. This is consistent with human observations inflammatory arthritis [5] induced by immune checkpoint inhibitors that was treated successfully with TNF- $\alpha$ inhibitors. In the genetically susceptible animal model, not only did blocking TNF- $\alpha$ reduce colitis but it also increased the anti-tumor activity of anticytotoxic T lymphocyte-associated antigen-4 and PD-1 antibodies. Therefore, if moderate or severe psoriatic lesions develop during nivolumab therapy, SSZ therapy cannot only treat the skin lesions but may also increase nivolumab's anti-tumor effect.

If dermatologic side effects occuring in patients receiving checkpoint inhibitor therapy that are not severe, therapy should not be stopped. For the treatment of moderate and severe psoriatic conditions during nivolumab therapy, we recommend SSZ, as it induces minimal side effects and may allow doses of nivolumab to be safely increased.

\section{Ethics}

Informed Consent: Consent form was filled out by all participants.

Peer-review: Internally peer-reviewed.

\section{Authorship Contributions}

Surgical and Medical Practices: G.O., K.N.P., C.D., Concept: G.O., K.N.P., C.D., Design: G.O., K.N.P., C.D., Data Collection or Processing: G.O., K.N.P., C.D., Analysis or Interpretation: G.O., K.N.P., C.D., Literature Search: G.O., K.N.P., C.D., Writing: G.O., K.N.P., C.D.

Conflict of Interest: No conflict of interest was declared by the authors.

Financial Disclosure: The authors declared that this study received no financial support.

\section{References}

1. Panariello L, Fattore D, Annunziata MC, Piantedosi F, Gilli M, Fabbrocini G. Bullous pemphigoid and nivolumab: dermatologic management to support and continue oncologic therapy. Eur J Cancer 2018;103:284-286.

2. Sibaud V, Meyer N, Lamant L, Vigarios E, Mazieres J, Delord JP. Dermatologic complications of anti-PD-1/PD-L1 immune checkpoint antibodies. Curr Opin Oncol 2016;28:254-263.

3. El-Mofty M, El-Darouti M, Rasheed H, Bassiouny DA, Abdel-Halim M, Zaki NS, el-Hanafy G, el-Hadidi H, Azzam O, el-Ramly A, Fawzy M. Sulfasalazine and pentoxifylline in psoriasis: A possible safe alternative. J Dermatolog Treat 2011;22:31-37.

4. Perez-Ruiz E, Minute L, Otano I, Alvarez M, Ochoa MC, Belsue V, de Andrea C, Rodriguez-Ruiz ME, Perez-Gracia JL, Marquez-Rodas I, Llacer C, Alvarez M, de Luque V, Molina C, Teijeira A, Berraondo P, Melero I. Prophylactic TNF Blockade Uncouples Efficacy and Toxicity in Dual CTLA-4 and PD-1 Immunotherapy. Nature 2019;569:428-432.

5. Cappelli LC, Gutierrez AK, Baer AN, Albayda J, Manno RL, Haque U, Lipson EJ, Bleich KB, Shah AA, Naidoo J, Brahmer JR, Le D, Bingham CO. Inflammatory arthritis and sicca syndrome induced by nivolumab and ipilimumab. Ann Rheum Dis 2017;76:43-50. 